\title{
FFT-ApEn Analysis for the Vibration Signal of a Rotating Motor
}

\author{
Yiqing Zhou \\ Department of Electronics and Information Technology, Jiangmen Polytechnic, Guangdong, PR China, 529090.
}

\author{
Shiuan-Ho Chang* \\ College of Electronics and Electrical engineering, Zhaoqing University, Guangdong, PR China, 526061.
}

\author{
Shuguang Wu, Xiong You Cai and Lin Tang \\ Department of Electronics and Information Technology, Jiangmen Polytechnic, Guangdong, PR China, 529090.
}

\author{
Z. Y. Xu \\ College of Electronics and Electrical engineering, Zhaoqing University, Guangdong, PR China, 526061.
}

\begin{abstract}
(Received 6 July 2017; accepted 26 January 2018)
This paper presents the FFT-ApEn analysis method for the fault detection of an electric motor under different rotating speeds. Motor vibration signals are analyzed using the Fast Fourier Transform (FFT) and Approximate Entropy (ApEn) to obtain the fault factor of a motor under different rotating speeds. The effectiveness of the proposed FFT and FFT-ApEn analyses for predicting the fault is verified through the experimental data. It is found that the FFT-ApEn analysis for the vibration signal can more precisely identify the fault as compared to the conventional FFT analysis method. In addition, the magnitudes of the frequency components are extracted for the recognition of the fault modes. The frequency spectrum analysis is used for distinguishing four operating statuses, i.e., normal, carbon brush failure, abnormal noise and bearing failure. Moreover, the FFT-ApEn method can successfully discriminate four different operating statuses of a motor without removing any motor parts. Hence, the FFT-ApEn analysis method is of great significance for a motor to have a real-time monitoring ability.
\end{abstract}

\section{INTRODUCTION}

A motor is an indispensable component in the modern industry. The temperature, noise, voltage, current and vibration signals are the most common parameters for detecting whether the status of a motor is normal or faulty1. Among all state-monitoring techniques, the vibration signal analysis is the most classical technique to evaluate the status of a rotating machine. $^{2-4}$ The analysis of a vibration signal is very critical to effectively monitor and control if a rotating motor possessed high productivity and reliability. Generally, for a fault diagnosis technique, the characteristic features of vibration signals were analyzed in frequency domain or chaotic phase space. For examples, Arslan et al. ${ }^{5}$ disclosed the relationship between statistical vibration parameters, tool wear, and surface roughness of a work piece during high speed turning operation. Javed et al. ${ }^{6}$ presented a method for feature extraction or selection, and the proposition was applied to time-frequency analysis of non-stationary signals using discrete wavelet transform. Zhang et al. ${ }^{7}$ used variational mode decomposition to detect the defect signals of different locations in a multistage centrifugal pump. They studied the failure mechanism of rolling bearings, and established the defect signal models of different locations, and simulated the fault signals of outer race defect, inner race defect, as well as the rolling element defect. Also, empirical mode decomposition (EMD) has been widely used for analyzing non-stationary signals due to its ability to self-adaptive decomposition of non-stationary signals. The EMD method can accurately identify and diagnose the running state and bearing

${ }^{*}$ Corresponding author. fault type at early stages of their development. ${ }^{8-10}$ Generally speaking, for a motion system, the complexity and chaos degree can be described by the Approximate Entropy (ApEn). The higher the complexity and chaos degree of a motion system are, the bigger the ApEn value of the system becomes. The ApEn analysis method has a strong ability of resisting noise interference in random signals, deterministic signals or these two mixed signals. Since this method was proposed, it has been successfully applied to the analyses of heart rate variability ${ }^{10}$ and endocrine hormone release pulsatility. ${ }^{12}$ Sparacino et al. ${ }^{13}$ studied a distorted portrait of the secretion rate at the gland level by ApEn analysis. They reported whether and how this distortion can influence the regularity of hormone pulsatility. On the other hand, in conventional condition monitoring, the commonly used method is the vibration analysis in frequency domain through Fast Fourier Transform (FFT). ${ }^{14}$ FFT is an algorithm to realize discrete Fourier transform and able to convert the vibration signal from its time- domain representation to its equivalent frequency-domain representation. Gao et al. ${ }^{15}$ presented an algorithm, FFT-AFD (Adaptive Fourier Decomposition), reducing the computational complexity of the AFD. AFD is originated with the purpose of positive frequency decomposition of signals. They have proven the effectiveness, accuracy, and reliability of the FFT-AFD algorithm, as well as laid a foundation for its practical applications. Nevertheless, frequency analysis is only one aspect of interpreting the information contained in a vibration signal. In view of this, this paper presents a FFT- ApEn method for the fault detection of a motor on the basis of both the chaotic space and frequency-domain analysis of vibration signals at different ro- 
tating speeds. It is found that the FFT-ApEn can more precisely identify the faulty type of a rotating motor as compared to the only FFT or ApEn method.

\section{RESEARCH METHOD}

\subsection{The Definition of the ApEn}

In order to define $\operatorname{ApEn}(r, m, N)$ for the N-dimensional time series $\{u(1), u(2), \ldots, u(N)\}$ with given parameters $m$ and $r$, the $m$-dimensional sequence vector $x(i)$ should be embedded. ${ }^{1,11}$ Then, the ApEn is defined as:

$$
\operatorname{ApEn}(m, r, N)=\lim _{N \rightarrow \infty}\left|\phi^{m}(r)-\phi^{m+1}(r)\right|
$$

where:

$$
\begin{gathered}
\varphi^{m}(r)=\left(\frac{1}{N-m+1}\right) \sum_{i=1}^{N-m+1} \ln C_{i}^{m}(r) ; \\
C_{i}^{m}(r)=\left(\frac{1}{N-m+1}\right) \sum_{j=1}^{N-m+1} \theta(d(x(i), x(j))-r), \\
\quad(1 \leq i \leq N-m+1, \quad i \neq j) \\
\theta(x)=\left\{\begin{array}{l}
1 \quad \text { if } x>0 \\
0 \quad \text { otherwise }
\end{array} ;\right. \\
d(x(i), x(j))=\max _{0 \leq k \leq m-1}|x(i+k)-x(j+k)|, \\
(1 \leq i \leq N-m+1,1 \leq j \leq N-m+1, i \neq j) .
\end{gathered}
$$

Obviously, the estimate value of the ApEn depends on $m$ and $r$. As suggested by Pincus et al., ${ }^{11} m$ can be taken as 2 and $r$ be taken as $(0.1-0.25) \mathrm{SD}$, where SD is the Standard Deviation from the original data sequence. As a rule in engineering practice, more than 100 data are needed to meet the requirements for estimating a robust value of the ApEn. Consequently in this paper, the ApEn is calculated under the following conditions: $N \geq 100, r=0.15 \mathrm{SD}, m=2$.

\subsection{The FFT-ApEn Analysis Method}

Seven motors that have been judged as four operating statuses were tested to obtain four sets of vibration signals, i.e., "normal" for two motors, "bearing failure" for two motors, "carbon brush failure" for two motors, and "abnormal sound" for a motor. The motors are series motors of the drum washing machines, and the DC voltage of $0-72 \mathrm{~V}$ is applied to them to get various rotating speeds.

Step 1: Acquiring the vibration signal for every typical operating status of a motor, and the frequency range of the vibration signal is divided into five equal regions.

Step 2: Through the FFT algorithm, ${ }^{15-17}$ computing the main frequency regions of the vibration signal for every operating status of the motor at low and high rotating speeds, respectively.

Step 3: Constructing the time series required for the calculation of the ApEn.

Step 4: Confirming the SD threshold $\delta_{0}$ and average-value threshold $\Psi_{0}$ of the ApEn for a normal-status rotating motor

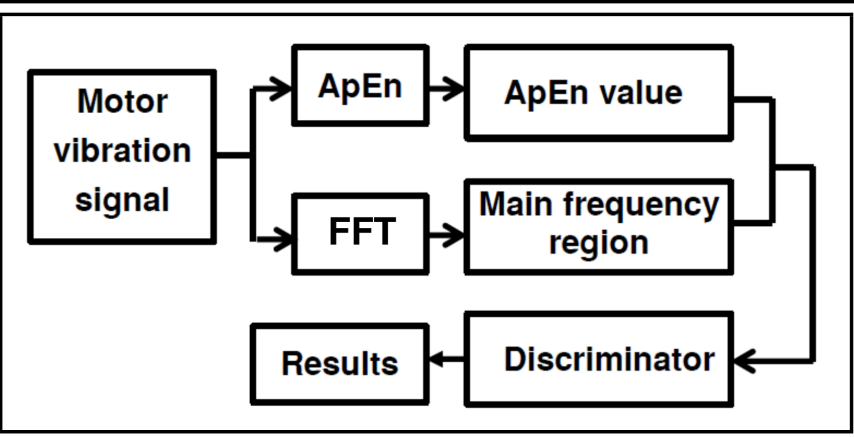

Figure 1. The flowchart of the FFT-ApEn algorithm



Figure 2. The flowchart of the decimation-in-time FFT algorithm $(N=8$.

at various rotating speeds.

Step 5: The SD and average value of the ApEn of the vibration signal for every operating motor are calculated and compared with $\delta_{0}$ and $\Psi_{0}$, at various rotating speeds. The flowcharts for the description of steps are revealed in Fig. 1 and 2.

\section{RESULTS AND DISCUSSION}

\subsection{Vibration Signals in Time and Frequency Domains}

As mentioned above, seven motors that have been judged as four operating statuses were tested to obtain four sets of vibration signals. The hardware components of the data acquisition system are Lens LC0105 accelerometer and NI9235 data acquisition card, as revealed in Fig. 3. Vibration signals of the motors in time or frequency domain at the rotating speeds of 915 and $2250 \mathrm{rpm}$ are shown in Figs. 4, 5, 6, and 7, respectively. The rotating speed of a motor is obtained from the location feedback by a photoelectric code-disc located at the motor revolution axis. From the vibration signals in time domain as seen in the Figs. 4 and 5, the four operating statuses have no obvious distortion phenomenon, so that it is difficult to evaluate the running states of the motors. Through the FFT algorithm, the vibration signals in frequency domain present obvious and different main frequency regions for the four operating-status motors at the same rotating speed of $915 \mathrm{rpm}$, as seen in the Fig. 6.

\subsection{Analysis of FFT Algorithm}

According to the vibration signal in frequency domain as shown in Fig. 6 and 7, the frequency range of the vibration signal for a motor is divided into five regions: $(0,500),(500$, $1000),(1000,1500),(1000,1500),(1500,2000),(2000,2500)$. By use of the FFT algorithm, main frequency regions of vi- 


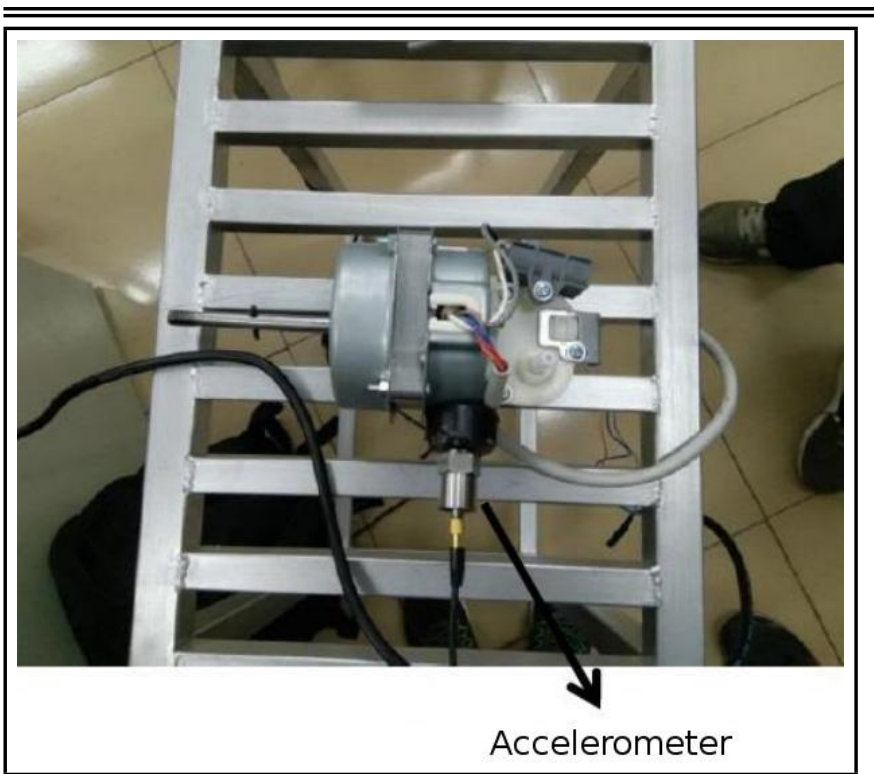

Figure 3. The picture of the experimental set up.

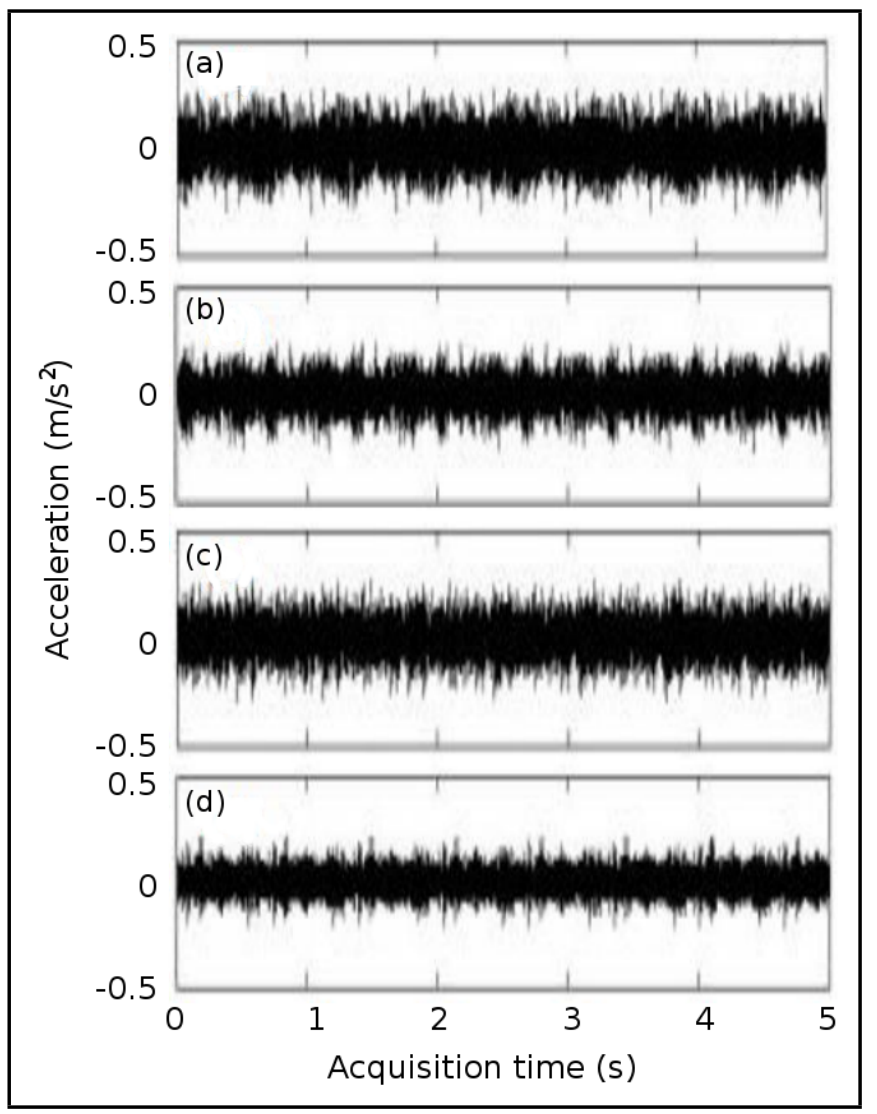

Figure 4. Vibration signals in time domain for four operating-status motors at the same rotating speed of $915 \mathrm{rpm}$. (a) normal (b) bearing failure (c) abnormal (d) carbon brush failure.

bration signals are partially overlapped for the four operatingstatus motors at a low rotating speed of $915 \mathrm{rpm}$, as shown in Fig. 6 and Table 1. Besides, the main frequency regions tend to be consistent for the four motors at a high rotating speed of 2250 rpm, as revealed in Fig. 7.

\subsection{The Average Value and Standard Deviation of the ApEn}

Table 2 shows the average value (AV) and standard deviation of the ApEn of the vibration signal for every operating-

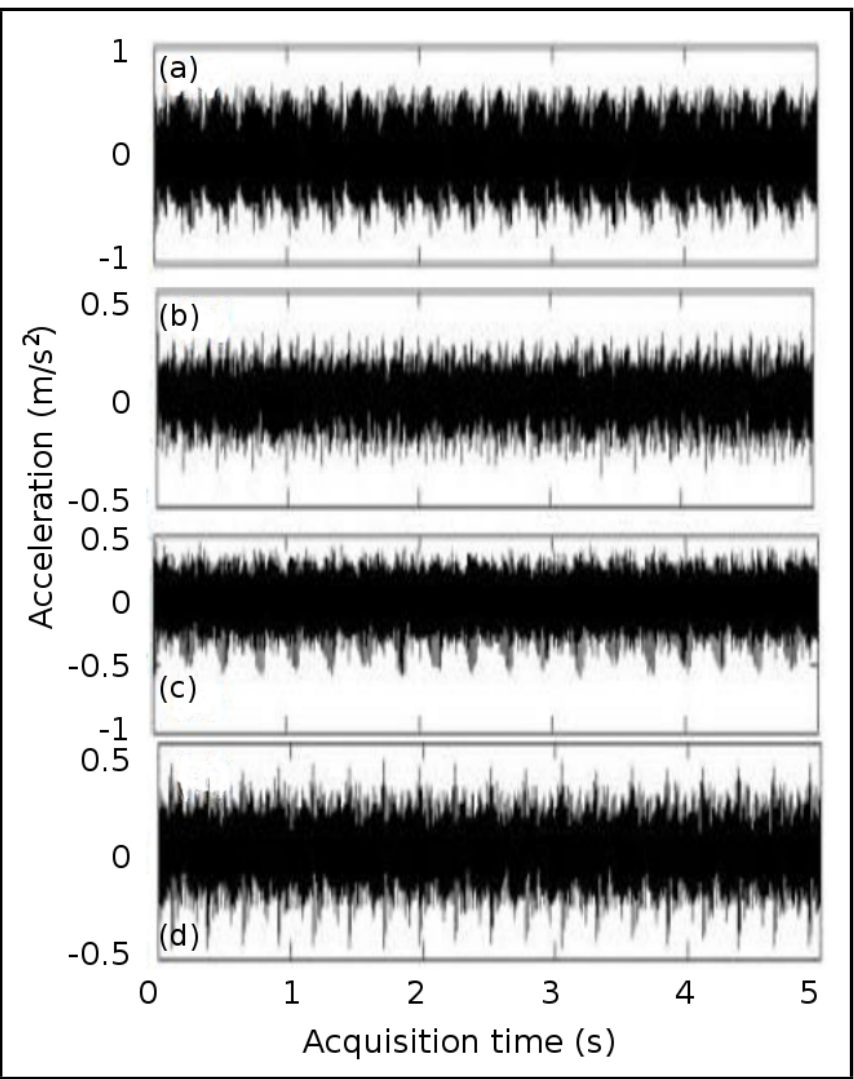

Figure 5. Vibration signals in time domain for four operating-status motors at the same rotating speed of $2250 \mathrm{rpm}$. (a) normal (b) bearing failure (c) abnormal (d) carbon brush failure.

Table 1. Main frequency features of vibration signals for four operating-status motors at different rotating speeds.

\begin{tabular}{||c|c|c|}
\hline Motor speed & Main frequency regions (Hz) & Operating status \\
\hline $915 \mathrm{rpm}$ & $(500,1000),(2000,2500)$ & normal \\
\hline $915 \mathrm{rpm}$ & $(500,1000)$ & bearing failure \\
\hline $915 \mathrm{rpm}$ & $(1500,2000),(2000,2500)$ & abnormal sound \\
\hline $915 \mathrm{rpm}$ & $(500,1000),(2000,2500)$ & carbon brush failure \\
\hline $2250 \mathrm{rpm}$ & $(1000,1500)$ & normal \\
\hline $2250 \mathrm{rpm}$ & $(1000,1500)$ & bearing failure \\
\hline $2250 \mathrm{rpm}$ & $(1000,1500)$ & abnormal sound \\
\hline $2250 \mathrm{rpm}$ & $(1000,1500)$ & carbon brush failure \\
\hline
\end{tabular}

status motor at different rotating speeds. From Table 2, the $\mathrm{AV}$ and SD of the ApEn of the normal motor are larger than those of the other faulty motors under the same rotating speed. When the motor rotating speed gets faster, the AV and SD differences between a normal motor and a faulty motor become larger. Therefore, comparing the AV and SD of the ApEn of a tested motor with those of a normal motor can judge if the tested motor was running normally. Unfortunately, when the $\mathrm{AV}$ and SD of the ApEn of a tested motor have been calculated out, it is difficult to discern the specific fault type by referring to Table 2 only.

\subsection{Judging the Operating Status of a Motor by FFT-ApEn Analysis}

To illustrate the FFT-ApEn method clearly, one normal motor (labeled as A) and three faulty motors (labeled as B, C, D) are tested as follows. Firstly, vibration signals are analyzed by ApEn algorithm for the four motors at a high rotating speed of $2250 \mathrm{rpm}$. Then, the operating status of every motor is evaluated as normal or not, as revealed in Table 3. Next, vibration signals are analyzed through the FFT algorithm for the four 


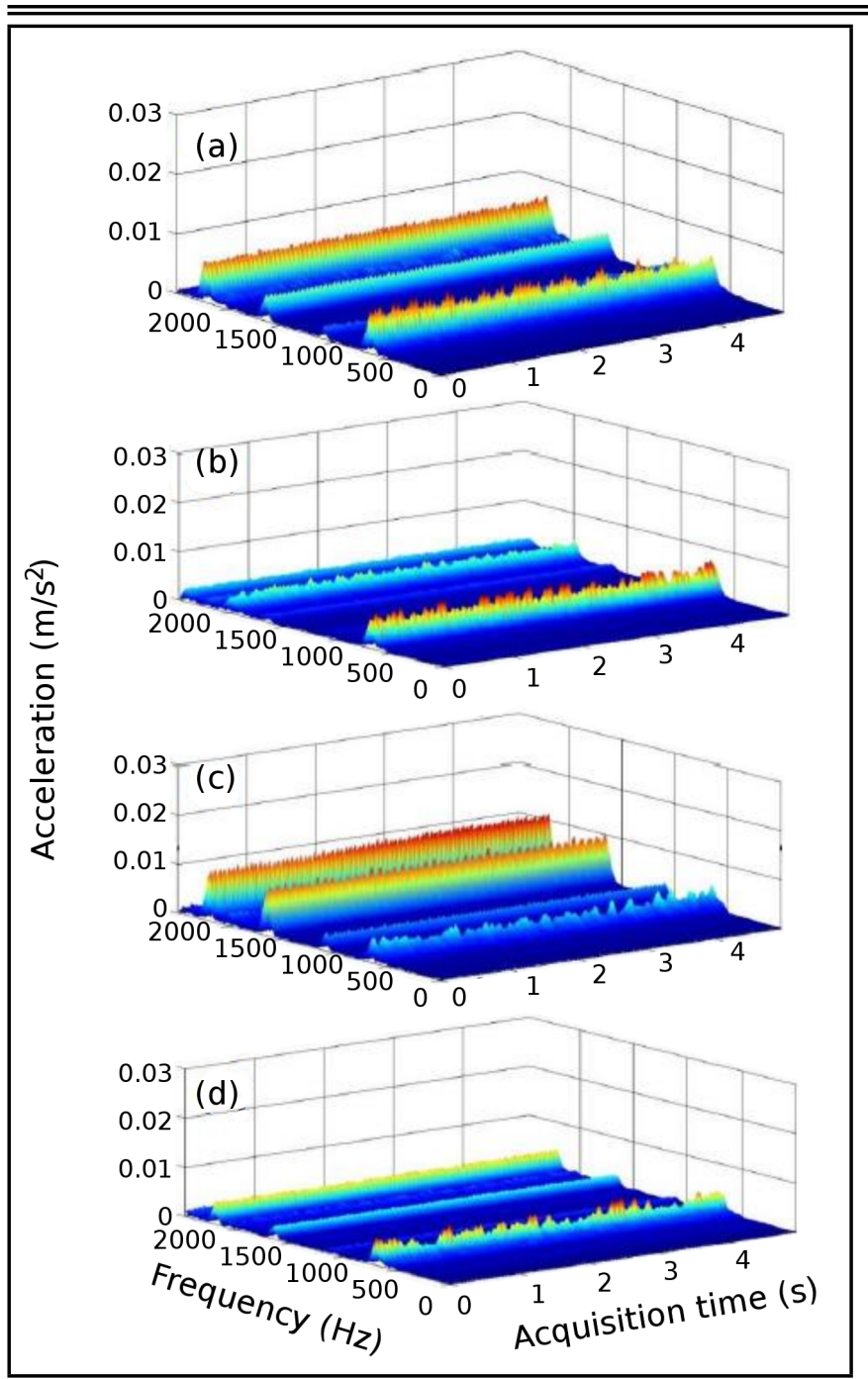

Figure 6. Vibration signals in frequency domain for four operating- status motors at the same rotating speed of $915 \mathrm{rpm}$. (a) normal (b) bearing failure (c) abnormal (d) carbon brush failure.

motors at a low rotating speed of $915 \mathrm{rpm}$, and the main frequency regions are extracted out. Finally, refering to Table 1 and Table 2, the operating status of every motor can be judged clearly, as shown in Table 4. For example, the AV (ApEn) of tested motor B is 0.0621087 at a rotating speed of $2250 \mathrm{rpm}$, as shown in Table 3. Refering to Table 2, the operating status of motor B could be either bearing or carbon brush failure. The main frequency of tested motor B is only $(500,1000)$ at a rotating speed of $915 \mathrm{rpm}$, as shown in Table 4. Then, refering to Table 1 , the operating status of motor B is judged as bearing failure.

\subsection{Discussion About Nonlinearity and Chaos}

By using the FFT algorithm, main frequency regions of the vibration signal for a motor are found out. According to the principle of nonlinear dynamics, the time series of variables already contain information about the system variables. In order to quantify the complexity of nonlinear sequence data, Pincus $^{18}$ proposed a concept of the ApEn, which solved the puzzle of getting the entropy of a chaotic system. Approximate entropy can be used to represent the complexity of a system, and it is widely used in the fields of the weather forecast and detection of the mechanical vibration. ${ }^{19}$

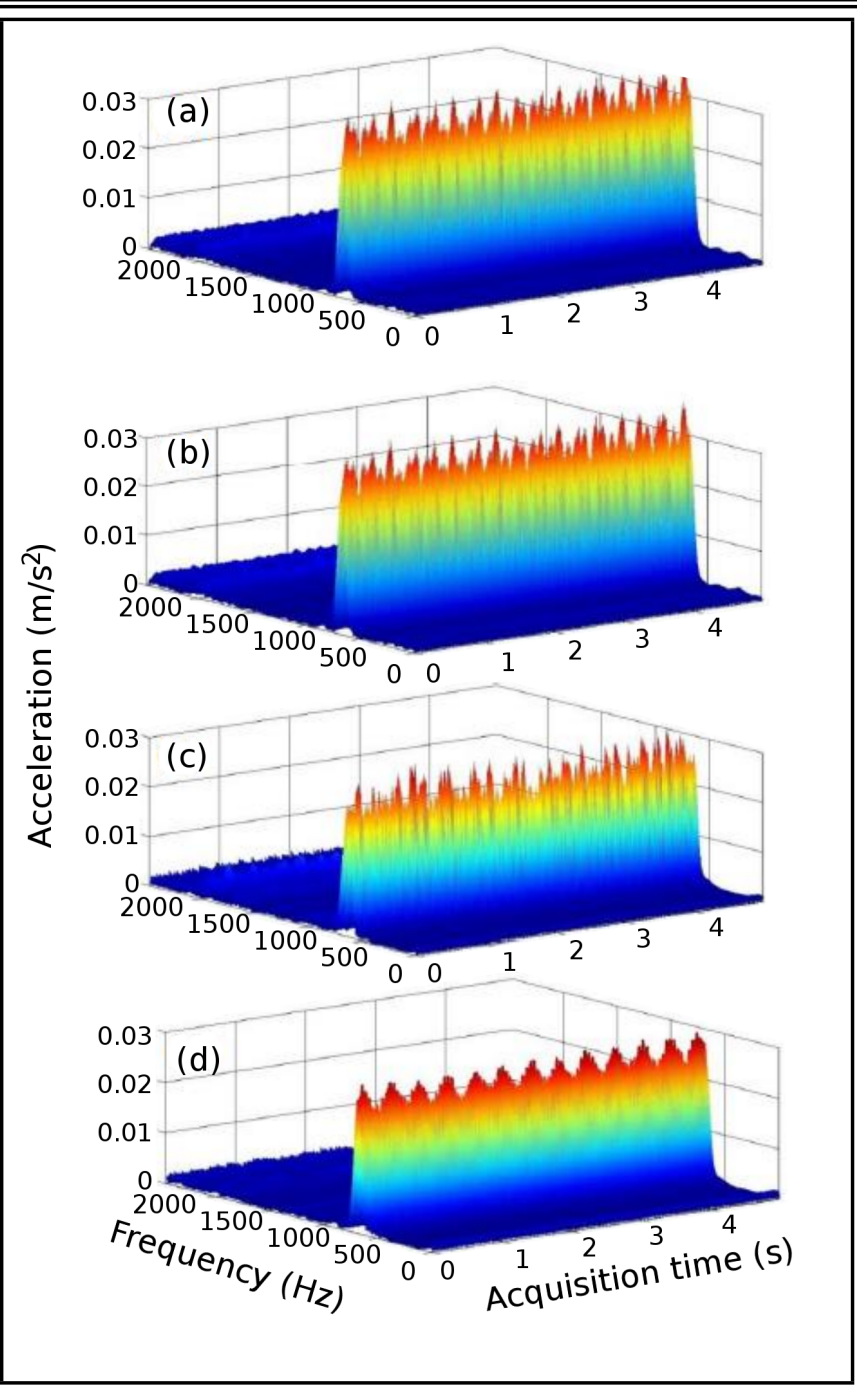

Figure 7. Vibration signals in frequency domain for four operating- status motors at the same rotating speed of $2250 \mathrm{rpm}$. (a) normal (b) bearing failure (c) abnormal (d) carbon brush failure.

\section{CONCLUSIONS}

Using FFT-ApEn analysis for a vibration signal, we investigated the relationship between the ApEn and stability of a rotating motor through the viewpoint of nonlinearity and chaos. The average value and standard deviation of the ApEn are significantly correlated with the operating status of a motor. For a normal motor, the value of the ApEn is increasing with the increasing of the rotating speed. To sum up, the FFT-ApEn method can successfully distinguish the operating status and is of great significance for a motor to have a real-time monitoring ability.

\section{ACKNOWLEDGEMENTS}

This work was supported by Pdjh2016b0752 and Jiangmen Science and Technology Project.

\section{REFERENCES}

1 Peŕez-Canales, D., Alvarez-Ramŕez, J., JaŕeguiCorrea, J.C., Vela-Martńez, L., Herrera-Ruiz, G. Identification of dynamic instabilities in machining process using the approximate entropy method, Int. J. Mach. Tool. Manu., 51, 556-564, (2011) https://dx.doi.org/10.1016/j.ijmachtools.2011.02.004 
Table 2. The average value and standard deviation of the ApEn for four operating-status motors at different rotating speeds.

\begin{tabular}{|c|c|c|c|}
\hline Motor speed & Operating status & AV (ApEn) & SD (ApEn) \\
\hline $915 \mathrm{rpm}$ & normal & $\mathbf{0 . 0 4 4 5 8 3 4}$ & $\mathbf{0 . 0 1 6 3 4 9 2}$ \\
\hline $915 \mathrm{rpm}$ & bearing failure & 0.0296041 & 0.0110936 \\
\hline $915 \mathrm{rpm}$ & abnormal sound & 0.0410724 & 0.0120309 \\
\hline $915 \mathrm{rpm}$ & carbon brush failure & 0.0175405 & 0.00861886 \\
\hline $1200 \mathrm{rpm}$ & normal & $\mathbf{0 . 1 5 4 3 2 6}$ & $\mathbf{0 . 0 2 2 5 3 9 7}$ \\
\hline $1200 \mathrm{rpm}$ & bearing failure & 0.0348034 & 0.0120916 \\
\hline $1200 \mathrm{rpm}$ & abnormal sound & 0.0283015 & 0.0100666 \\
\hline $1200 \mathrm{rpm}$ & carbon brush failure & 0.0175405 & 0.00861886 \\
\hline $1700 \mathrm{rpm}$ & normal & $\mathbf{0 . 3 0 5 2 7 9}$ & $\mathbf{0 . 0 4 6 5 3 3 2}$ \\
\hline $1700 \mathrm{rpm}$ & bearing failure & 0.100699 & 0.0169607 \\
\hline $1700 \mathrm{rpm}$ & abnormal sound & 0.0818955 & 0.0182974 \\
\hline $1700 \mathrm{rpm}$ & carbon brush failure & 0.0544811 & 0.0181129 \\
\hline $2250 \mathrm{rpm}$ & normal & $\mathbf{0 . 3 2 0 2 1 6}$ & $\mathbf{0 . 0 4 6 0 2}$ \\
\hline $2250 \mathrm{rpm}$ & bearing failure & 0.0625301 & 0.0287796 \\
\hline $2250 \mathrm{rpm}$ & abnormal sound & 0.154206 & 0.013487 \\
\hline $2250 \mathrm{rpm}$ & carbon brush failure & 0.0748915 & 0.0186085 \\
\hline
\end{tabular}

Table 3. The AV and SD of the ApEn for four tested motors at a high rotating speed of $2250 \mathrm{rpm}$

\begin{tabular}{|c|c|c|c|}
\hline $\begin{array}{c}\text { Tested motor } \\
\text { (2250 rpm) }\end{array}$ & $\begin{array}{c}\text { AV } \\
\text { (ApEn) }\end{array}$ & $\begin{array}{c}\text { SD } \\
\text { (ApEn) }\end{array}$ & $\begin{array}{c}\text { Operating } \\
\text { status }\end{array}$ \\
\hline A & $\mathbf{0 . 3 2 0 7 2 7}$ & $\mathbf{0 . 0 4 5 8 8}$ & normal \\
\hline B & 0.0621087 & 0.0296798 & abnormal \\
\hline C & 0.151106 & 0.013502 & abnormal \\
\hline D & 0.0752915 & 0.0185985 & abnormal \\
\hline
\end{tabular}

Table 4. Main frequency features for four tested motors at a low rotating speed of $915 \mathrm{rpm}$.

\begin{tabular}{|c|c|c|}
\hline $\begin{array}{c}\text { Tested motor } \\
(\mathbf{9 1 5} \mathbf{~ r p m})\end{array}$ & $\begin{array}{c}\text { Main frequency } \\
(\mathbf{H z})\end{array}$ & $\begin{array}{c}\text { Operating } \\
\text { status }\end{array}$ \\
\hline $\mathrm{A}$ & $(500,1000),(2000,2500)$ & normal \\
\hline $\mathrm{B}$ & $(500,1000)$ & bearing failure \\
\hline $\mathrm{C}$ & $(1500,2000),(2000,2500)$ & abnormal sound \\
\hline $\mathrm{D}$ & $(500,1000),(2000,2500)$ & carbon brush failure \\
\hline
\end{tabular}

2 Sakthivel N. R., Sugumaran V., Babudevasenapati S., Vibration based fault diagnosis of monoblock centrifugal pump using decision tree, Expert Syst. Appl., 37, 4040-4049, (2010) https://dx.doi.org/10.1016/j.eswa.2009.10.002

3 Zhao, H.. Deng, W., Yang, X., Li, X. Research on a vibration signal analysis method for motor bearing, Optik-International Journal for Light and Electron Optics, 127, 10014-10023, (2016) https://dx.doi.org/10.1016/j.ijleo.2016.07.046

4 Kumar, A., Kumar, R. Time-frequency analysis and support vector machine in automatic detection of defect from vibration signal of centrifugal pump, Measurement, 108, 119-133, (2017) https://dx.doi.org/10.1016/j.measurement.2017.04.041

5 Arslan, H., Er, A. O., Orhan S., Aslan, E. Tool Condition Monitoring in Turning Using Statistical Parameters of Vibration Signal, Int. J. Acousti. Vib., 21 (4), 371-378, (2016) https://dx.doi.org/10.20855/ijav.2016.21.4432

6 Javed, K., Gouriveau, R., Zerhouni, N., Nectoux, P. Enabling Health Monitoring Approach Based on Vibration Data for Accurate Prognostics, IEEE Trans. Ind. Electron., 62, 647-656, (2014) https://dx.doi.org/10.1109/TIE.2014.2327917

7 Zhang, M., Jiang, Z., Feng, K. Research on variational mode decomposition in rolling bearings fault diagnosis of the multistage centrifugal pump, Mech. Syst. Signal Pr., 93, 460-493, (2017) https://dx.doi.org/10.1016/j.ymssp.2017.02.013

${ }^{8}$ Dong, H., Qi, K., Chen, X., Zi, Y., He, Z., Li, B. Sifting process of EMD and its application in rolling element bearing fault diagnosis. J. Mech. Sci. Technol., 23, 2000-2007, (2009) https://dx.doi.org/10.1007/s12206-009-0438-9

${ }^{9}$ Wang K. S., Heyns P. S., Application of computed order tracking, Vold-Kalman filtering and EMD in rotating machine vibration, Mech. Syst. Signal Pr., 25, 416-430, (2011) https://dx.doi.org/10.1016/j.ymssp.2010.09.003

${ }^{10}$ Dybała, J., Zimroz, R. Rolling bearing diagnosing method based on empirical mode decomposition of machine vibration signal, Appl. Acoust., 77, 195-203, (2014) https://dx.doi.org/10.1016/j.apacoust.2013.09.001

11 Pincus, S. M., Goldberger, A. L., Physiological Time-series Analysis: What Does Regularity Quantify? Am. J. Physiol., 266, 1643-1656, (1994) https://dx.doi.org/10.1152/ajpheart.1994.266.4.H1643

12 Pincus, S., Mulligan, T., Iranmanesh, A., Gheorghiu, S., Godschalk, M., Veldhuis, J. Older males secrete luteinizing hormone and testosterone more irregularly, and jointly more asynchronously, than younger males, Proc. Natl. Acad. Sci. USA, 93, 14100-14105, (1996) https://dx.doi.org/10.1073/pnas.93.24.14100

13 Sparacino, G., Bardi, F., Cobelli, C. Approximate Entropy Studies of Hormone Pulsatility from Plasma Concentration Time Series: Influence of the Kinetics Assessed by Simulation, Ann. Biomed. Eng., 28, 665-676, (2000) https://dx.doi.org/10.1114/1.1306344

${ }^{14}$ Farokhzad, S. Vibration Based Fault Detection of Centrifugal Pump by Fast Fourier Transform and Adaptive NeuroFuzzy Inference System, J. Mech. Eng. Technol., 1(3), 8287, (2013) https://dx.doi.org/10.18005/JMET0103001

15 Gao, Y., Ku, M., Qian, T., Wang, J. FFT formulations of adaptive Fourier decomposition, J. Comput. Appl. Math., 324, 204-215, (2017) https://dx.doi.org/10.1016/j.cam.2017.04.029

16 Marichal, G. N., Arts, M., Garca-Prada, J. C., An intelligent system for faulty-bearing detection based on vibration spectra, J. Vib. Control, 17, 931-942, (2011) https://dx.doi.org/10.1177/1077546310366264

17 Lilo, M. A., Latiff, L. A., Al Mashhadany, Y. I., Abu, A. B. H. Identify and Classify Vibration Signal for Steam Turbine Based on Neural Sleep Fuzzy System, Res. J. Appl. Sci. Eng. Techno., 12, 589-598, (2016), https://dx.doi.org/10.19026/rjaset.12.2687

18 Pincus, S. M. Approximate entropy as a measure of system complexity, Proc. Nati. Acad. Sci. USA, 88, 2297-2301, (1991) https://dx.doi.org/10.1073/pnas.88.6.2297

19 Lorenz, E. N. Deterministic nonperiodic flow, J. Atmos. Sci., 20, 130-141, (1963) https://dx.doi.org/10.1175/15200469(1963)020<0130:DNF $>2.0 . \mathrm{CO} ; 2$ 